\title{
Development of an Implementation Framework for Overcoming Underdiagnoses of Familial Hypercholesterolemia in the USA
}

\author{
Dana M. Miller ${ }^{a} \quad$ Amy Gaviglio ${ }^{b}$ Heather A. Zierhut ${ }^{a}$ \\ aDepartment of Genetics, Cell Biology, and Development, University of Minnesota, Minneapolis, MN, USA; \\ ${ }^{b}$ G2S Corporation/CDC Newborn Screening and Molecular Biology Branch, Atlanta, GA, USA
}

\section{Keywords}

Familial hypercholesterolemia - Genetic screening · Healthcare services · Implementation science · Public health · Public health genetics · Public health genomics

\begin{abstract}
Familial hypercholesterolemia $(\mathrm{FH})$ is a genetic condition which causes elevated low-density lipoprotein cholesterol from birth. With a prevalence of 1 in 250 and the availability of effective treatments, the diagnostic rate of $<1$ to $10 \%$ is unacceptably low. Screening for $\mathrm{FH}$ is supported by multiple organizations, but it has not been broadly adopted and implemented across the USA. To investigate the implementation of FH screening, key informants were recruited from across the USA for their expertise in FH-related literature, guidelines, public health, and/or advocacy to complete semistructured interviews guided by implementation science (RE-AIM framework). Sixteen semistructured interviews were analyzed with directed content and thematic analyses, yielding specific barriers and recommendations to improve $\mathrm{FH}$ screening. Barriers to $\mathrm{FH}$ screening included patient recruitment and participation, equitable access to healthcare, provider discomfort with screening and treating $\mathrm{FH}$, provider burden, lack of public health and legislative support, $\mathrm{FH}$ awareness, guideline complexity, facilitation of genetic test-
\end{abstract}

ing and cascade screening, and lack of coordination between stakeholders. Awareness, engagement, communication, and collaboration between stakeholders is integral to successful FH screening. Individualized plans will be required at national, regional, and institutional levels. FH screening implementation can be achieved through practice facilitation, streamlined screening approaches, electric medical record tools, and consensus guidelines to increase screening adoption and consistent delivery. Reliable funding and established lines of communication between stakeholders can maintain efforts as FH screening progresses.

(c) 2021 S. Karger AG, Basel

\section{Introduction}

Familial hypercholesterolemia (FH) is an autosomal dominant genetic condition in which affected individuals have significantly increased low-density lipoprotein cholesterol (LDL-C) from birth [1]. The clinical presentation of FH is variable due to genetic, metabolic, and environmental factors, but untreated individuals with $\mathrm{FH}$ have an increased risk of atherosclerotic cardiovascular disease and death $[2,3]$. Relative to other genetic conditions, $\mathrm{FH}$ is common, affecting 1 in 250 individuals across all genders and ethnic groups $[3,4]$. Despite the prevalence, $\mathrm{FH}$ 
remains severely underdiagnosed, with diagnostic rates ranging from $<1$ to $10 \%$ [5]. In the absence of early screening programs, untreated individuals often present with atherosclerotic disease, but identification of presymptomatic affected individuals through screening facilitates early and effective treatment $[2,3,6-8]$.

FH screening has been supported by multiple public health organizations. The condition largely meets the Wilson and Jungner [9] criteria for a suitable screening condition and is a Centers for Disease Control and Prevention (CDC) tier 1 condition indicating significant potential for a positive impact on public health [10]. Professional guidelines sponsored by the National Heart, Lung, and Blood Institute (NHLBI) and adopted into policy by the American Academy of Pediatrics (AAP) recommend universal screening for $\mathrm{FH}$ in children, regardless of symptoms in 2011 [11]. The policy was updated to an AHA/ACC lipid guideline in 2018 [12]. For FH, universal screening generally uses a total serum cholesterol threshold to identify those who would benefit from further diagnostic testing $[2,11,13]$. Universal screening has the greatest potential benefit in younger, presymptomatic individuals when treatment is most beneficial [14]. However, the US Preventive Services Task Force (USPSTF) reports that there is still insufficient evidence concerning the diagnostic yield, treatment effectiveness, harms of screening, and changes in outcomes to adequately assess the benefits and harms of universal FH screening in children under 20 years of age $[11,15]$.

After a suspected FH case is identified, a clinical diagnosis of FH typically involves criteria such as significantly elevated total or LDL-cholesterol, physical findings, premature coronary artery disease, DNA analysis, and family history of the same [16]. These factors are integrated into FH diagnostic criteria such as the Simon Broome criteria, the Dutch Lipid Clinic criteria, or the US Make Early Diagnosis to Prevent Early Death (MEDPED) criteria [17]. A genetic diagnosis of FH requires the identification of a pathogenic variant in one of the genes known to cause FH (e.g., LDLR, APOB, and PCSK9) [18]. As cholesterol screening is not specific for $\mathrm{FH}$, diagnosis can be bolstered by genetic testing and facilitate diagnosis for at-risk family members $[3,18-23]$. Cascade screening is often delayed or does not occur due to complicated family dynamics; however, medical professional assistance can increase cascade screening uptake [24-26].

Healthcare providers have historically been the gateway to universal FH screening in the USA, yet many are still unfamiliar with existing guidelines and their specific role in the overall process. Primary care physicians report

Implementation Framework for

Overcoming Underdiagnoses of FH a responsibility to identify $\mathrm{FH}$ and acknowledge early treatment reduces cardiovascular risk, but physicians, overall, do not incorporate screening into their practices $[27,28]$. Low rates of screening are in-part due to provider discomfort in management, opposition to the use of lipid drugs in children, and unfamiliarity with screening guidelines [28]. Approximately, a third of physicians provide no lipid screening, half screen selectively, and $16 \%$ screen universally [28]. Pediatricians are more likely to screen; however, as few as $4 \%$ screen all eligible patients $[28,29]$. Questions remain regarding how best to screen for FH in the USA to ensure consistently and effectively implemented programs.

Last, cost-effectiveness is an important consideration for screening programs $[14,30]$. Universal screening for $\mathrm{FH}$ is generally more expensive than cascade screening, but can be cost-effective if done in targeted groups such as children [14]. Cascade screening is most cost-effective if there is a known pathogenic variant in a family $[14,31$, 32 ]. Both screening modalities have unique benefits and have successfully applied in combination across various countries [13, 33-37].

The aim of our study was to elicit key informant (KI) opinions about barriers to $\mathrm{FH}$ screening and potential strategies to overcome them with the purpose of facilitating implementation of FH screening in the USA. To interrogate the implementation components, we used the RE-AIM framework. RE-AIM uses implementation science to present components needed to create, implement, and maintain a program or initiative, and it has been successfully applied in preventative medicine programs [3840]. The domains of the RE-AIM framework were applied to FH screening (Fig. 1). Addressing each domain individually builds a comprehensive view of the public health issue and allows for practical solutions.

\section{Methods}

\section{Recruitment}

KI interviews were chosen to collect qualitative perspectives from individuals with specialized knowledge relevant to $\mathrm{FH}$ screening implementation [41]. Recruitment of KIs took place between September 2018 and January 2019. A list of 35 individuals was initially created by D.M., H.Z., and A.G. for their expertise in FH or cardiology-related research, practice, public health efforts, or advocacy. Eligible KIs were English-speaking and fit into $3 \mathrm{KI}$ categories (public health, research and advocacy, or direct healthcare). KIs who completed the interview and KIs who declined the interview were asked to refer other KIs. Twenty-seven KIs were prioritized by D.M. and H.Z. based upon their positions, years of experience, and diverse perspectives. 
Fig. 1. The RE-AIM framework. The implementation science RE-AIM framework comprises five domains: reach, effectiveness, adoption, implementation, and maintenance. The definitions of each domain are provided within the context of familial hypercholesterolemia screening.

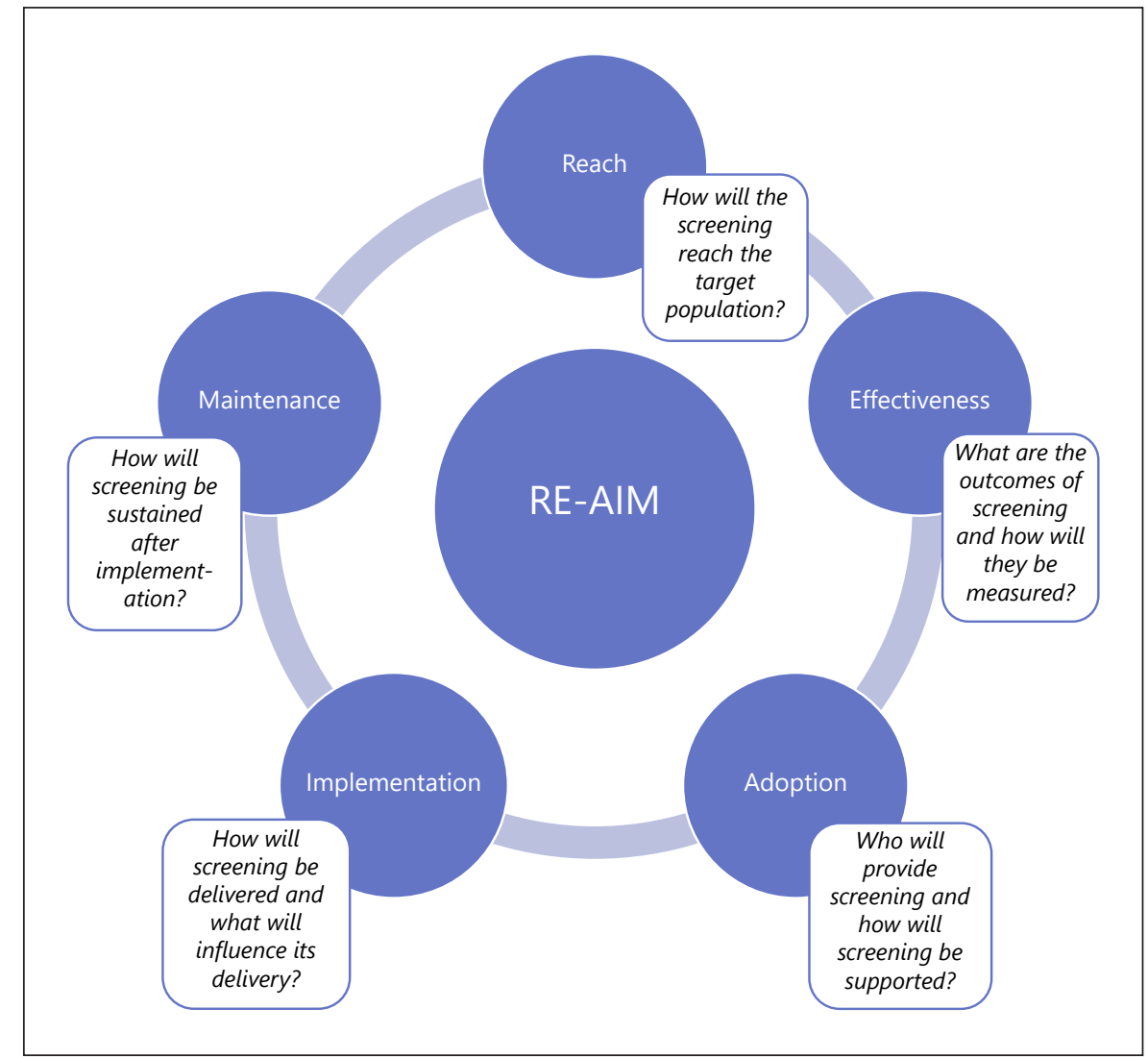

\section{Data Collection}

A semistructured interview guide, comprising 17 questions with prompts, was created to assess domains of the RE-AIM implementation framework (online suppl. material 1; for all online suppl. material, see www.karger.com/doi/10.1159/000513872). Questions were written to address policies and practices relevant to $\mathrm{FH}$ screening that could be broadly applied to the US healthcare system. Questions addressed successful strategies and barriers to FH screening and elicited suggestions for FH screening improvement and implementation. A.G. piloted the interview, and minor edits were made to create a final interview guide. Demographics collected included position, years of $\mathrm{FH}$ professional experience, and gender identity.

KIs were sent an invitation to participate in the interview via email, and were reminded via email 14 days later if no response was received. Interviews were conducted and audio recorded over telephone by D.M. between September 2018 and February 2019. Field notes were taken during interviews by D.M., and transcription was completed and audited by 3 independent transcribers. Demographic information about the researchers was shared with inquiring KIs, but none of the KIs had a prior relationship with the interviewer. Upon completion of interviews, KIs were compensated for their time with a USD 20 www.amazon.com gift card.

\section{Data Analysis}

Directed content analysis using a codebook based on the REAIM framework and its definitions was used for categorization of codes (Table 1) $[42,43]$. Interviews were coded by D.M. using NVivo software (version 12), and thematic analysis was used to create additional themes specific to $\mathrm{FH}$. Themes were aligned to the appropriate RE-AIM domain by D.M., coding was then reviewed by H.Z., and discrepancies were resolved in consultation with D.M. until consensus was reached. KI demographics were collected and summarized.

\section{Results}

\section{Demographics}

A total of $27 \mathrm{KIs}$ were contacted, 16 of whom responded to the recruitment email and completed an interview (public health, $n=6$; direct healthcare, $n=6$; research and advocacy, $n=4$ [Table 2]). All other informants neither responded nor completed interviews.

\section{RE-AIM Framework}

Using the RE-AIM framework, themes arose within each of the 5 domains. Themes were organized by the most relevant domain, but overlap did arise. The 3 most prominent domains were reach, adoption, and imple- 
Table 1. Codebook designed using the RE-AIM framework

\begin{tabular}{|c|c|c|}
\hline Code & Subcode & Description \\
\hline \multirow[t]{2}{*}{ Reach } & Target population & The demographics of the population who is screened \\
\hline & Recruitment & How to encourage the public to participate in screening \\
\hline \multirow[t]{2}{*}{ Effectiveness } & Potential positive effects of screening program & The benefits that could arise from an FH screening program \\
\hline & Potential negative effects of screening program & The issues that could arise from an FH screening program \\
\hline \multirow[t]{6}{*}{ Adoption } & Setting & The location of proposed screening and the region it would cover \\
\hline & Intervention agent & The proposed person to provide the initial screening \\
\hline & Provider participation & How to support and encourage providers to screen the target population \\
\hline & Follow-up intervention agent & The person who will follow-up on positive cases after initial screening is completed \\
\hline & Public health support and legislative support & How public health departments can support the implementation of screening \\
\hline & Research & What information is still needed to support implementation of screening \\
\hline \multirow{2}{*}{$\begin{array}{l}\text { Implemen- } \\
\text { tation }\end{array}$} & Consistency of delivery & How to provide screening to all members of the target population \\
\hline & Cost and funding of screening & Related to the cost of testing or screening (cholesterol screening and genetic testing) \\
\hline \multirow{2}{*}{ Maintenance } & Registries & The support of an FH registry, enrollment in the registry, and data utility \\
\hline & Consent and data privacy & $\begin{array}{l}\text { How to balance the participants' autonomy, informed consent, and privacy with public } \\
\text { benefit }\end{array}$ \\
\hline
\end{tabular}

The 5 domains were used to code the interviews with themes arising within each domain. Themes were created based on RE-AIM definitions and KI responses. FH, familial hypercholesterolemia; KI, key informant.

mentation. These domains were also the most highly variable in terms of KI responses. Themes were generally present across all informant groups unless otherwise noted.

\section{Reach}

KIs had varying opinions about the reach of an $\mathrm{FH}$ screening program. Themes included the population(s) to be targeted, how populations will be recruited, and the incentives and barriers that may influence participation in $\mathrm{FH}$ screening programs.

\section{Target Population}

Most KIs suggested that FH screening should occur during childhood, early adulthood, or both. KIs noted that narrow targeted age groups may miss a large portion of the population. A single screening approach was not expected to be successful across the entire country or state due to diverse medical institutions and differing population demographics. To screen the most individuals, diverse and redundant approaches for $\mathrm{FH}$ screening were suggested. Most KIs supported universal FH screening.

Implementation Framework for

Overcoming Underdiagnoses of FH

\section{Recruitment}

All KIs cited a need for increased public awareness to encourage public participation in $\mathrm{FH}$ screening programs. Multiple awareness and educational campaigns were suggested using social media, website banner ads, billboards, and/or celebrity endorsements. KIs recommended that awareness campaigns emphasize the differences between high cholesterol, in general, and FH. Campaigns should also dispel the myth that heart disease only affects the elderly. Direct healthcare KIs suggested increased public messaging from professional organizations and patient reminders from providers directly. Reminder letters sent to patients' homes were not expected to significantly increase $\mathrm{FH}$ screening unless there was an alarming text (e.g., bolded red font and strong language).

KIs mentioned logistical barriers to patient recruitment such as a reluctance to draw blood from a child, reluctance to participate in fasting cholesterol screens, and rushed primary care appointments. The majority of KIs suggested coupling FH screening with existing blood draws or immunizations to increase patient convenience. A few KIs suggested using school systems and some sug- 
dation Cascade Screening for Awareness and Detection (CASCADE) FH registry; however, many mentioned the need for state-level data interpretation. Some KIs advocated for wider access and use of registry data to improve utility. KIs suggested formal research studies on screening rates, screening methodology, cascade screening, diagnostic rates, treatment, adverse outcomes, and genotype-phenotype correlations.

\section{Consent and Data Privacy}

KIs also felt that patient consent regarding the privacy and use of registry data was important. KIs cited concerns over the privacy and security of the data, and specific concerns about patient insurability, employment, and discrimination were included. Most KIs felt that general privacy measures were adequate, and data privacy risk was acceptable considering the benefits. Over time, there should be data monitoring and management to ensure the data's integrity.

\section{Potential Positive Outcomes of Screening}

Nearly all KIs noted FH screening had the potential to increase diagnostic rates and decrease associated health outcomes. KIs across all groups cited the opportunity for early behavior modification, reduction in adverse cardiac events, and savings in healthcare costs.

One KI stated, "identify this at a young age and help prevent secondary outcomes. I mean that's going to be saving a lot of money in general... in healthcare costs." (KI13, direct healthcare, 2.5 years experience)

KIs also said that increased screening can spread public awareness of $\mathrm{FH}$, alter perceptions of heart health, and normalize FH for those that are diagnosed.

\section{Potential Negative Outcomes of Screening}

There were concerns about false-positive screens related to lifestyle factors burdening providers. Some KIs were fearful that statins would be prescribed to children who do not require the medication. To address unexpected findings and false positives, KIs suggested clear management guidelines with adjustments over time to mitigate any unexpected negative outcomes. Direct healthcare KIs thought that there may be a burden on the healthcare system potentially increasing wait times, but most KIs felt that the bulk of the care could be managed by the patient's primary care provider.

\section{Adoption}

KIs across all groups were interested in increasing adoption of a screening program, but there were many conflicting opinions within and between groups as to how the program should be envisioned.

\section{Setting}

KIs differed in their opinions about the scope of an FH screening program. Some KIs stated that a nation-wide program would be ideal but is not feasible due to separation between healthcare systems, issues with cross-institutional communication, and an inability to agree on screening initiatives across constituencies. The majority of KIs favored a state-wide or smaller, regional approach because they allow for program individualization. Statewide approaches to screening were favored by KIs in research/advocacy.

Direct healthcare KIs favored a smaller, regional approach to $\mathrm{FH}$ screening relative to state-wide and national approaches. Region approaches could be coordinated across local clinics or within larger medical institutions that serve multiple sites. "[T]here's different approaches that are going to be valid in different settings even within a single country- that one-size-fits-all approach is probably not ideal in any scenario. It's going to take many different approaches to identify as many FH patients as we need." (KI10, research/advocacy, 15 years experience)

\section{Intervention Agent}

There was wide agreement that either primary care physicians or pediatricians should be the intervention agent providing initial screening. Three KIs suggested that multiple providers should offer screening, and onequarter of KIs suggested a nation- or state-wide method such as a school program or a foundation-run program.

\section{Provider Participation}

All KIs recognized the importance of provider support and participation through increasing provider awareness and reducing provider discomfort. Suggestions included public health or provider educational outreach, grand rounds presentations, courses with educational credit, brochures, and websites. Provider awareness initiatives were suggested to take place gradually over time to increase their adoption.

All KI groups said that providers need a long-term, evidence-based plan for how to manage cases before they will feel comfortable with screening. Plans would include management of family members, information about where and when to refer, handouts, and treatment for both false-positive and true-positive cases. "Most pediatricians are very comfortable screening for hyperlipidemia, but I would say they're actually fair- 
ly uncomfortable managing it." (KI16, direct healthcare, 15 years experience)

Multiple research/advocacy KIs suggested using practice tools and websites to guide providers' case management. Many KIs were concerned about the extra time that would be required of providers as a result of increased $\mathrm{FH}$ screening and suggested providing clear steps to set up a program, notifications via electronic medical record prompts, and integrating screening into the clinic workflow. It may also involve implementing a program for providers in some cases: "[T]here needs to be some degree of clarity... if we're going to ask people to do the screening, [providers need] tools around how to easily and effectively...consistently implement it into their processes." (KI4, public health, 18.5 years experience)

Reducing barriers in implementing programs also included piggybacking screening with other tasks in their workflow. "Whatever can be done to lessen the burden on clinicians who are already overworked...so as long as there's compensation... and as long as it's something that can be well integrated into their workflow." (KI5, public health, unknown years experience)

Following screening, many KIs from all groups said the utility of screening must be demonstrated to providers. This could be shared using descriptive reports about diagnostic changes in the provider's area and success stories from screening.

All KI groups said appreciation for provider participation was crucial to keep providers vested in a screening program. Appreciation can be provided verbally from overseeing organizations, through monetary compensation for services, or incentives. Quality measures like the Minnesota Community Measures Recommendation or the National Quality Forum were mentioned as ways to increase consistent compensation. "[T]here's no incentive right now to do it. Except for if you're in a large healthcare delivery network for which you receive a bonus for optimal care...In medicine there are things we get penalized for in terms of insurance reimbursement... and at some point, incentives for FH or high cholesterol might come in." (KI10, research/advocacy, 15 years experience)

\section{Follow-Up Intervention Agent}

The majority of KIs suggested that a specialized $\mathrm{FH}$ team approach would be the most appropriate way to follow-up positive cases from initial screening. This would be led by primary care and include other specialties as needed such as cardiology, dyslipidemia, endocrinology, genetics, dietetics, or social work.
KIs were split as to whether they thought increased screening would overload specialty clinics. Regardless, KIs said that follow-up intervention agents should be comfortable managing patients and have the appropriate referral network in place; referrals should be available, individualized, and limited solely to what is necessary.

\section{Public Health and Legislative Support}

KIs within and between groups had differing opinions about how public health can support FH screening, suggesting public health support integration of screening into existing clinical workflows, partnerships with advocacy groups, and monetary support. KI opinions about monetary public health support varied significantly: suggestions included broad program funding, integration of FH into existing public health block grants, funding only for services that payers decline to cover, and funding for populations with reduced access to care. Other KIs suggested that public health's role should support providers in screening using the tools they already have. "[P]ractice facilitators are often trying to do is trying to help [clinics] overcome some of those challenges that may be in their way, so it's really more of a hands-on approach of, of encouraging more rapid change within organizations." (KI3, public health, 20 years experience)

KIs in direct healthcare and research/advocacy also mentioned that the lack of awareness of $\mathrm{FH}$ perpetuates a lack of screening. Despite FH's classification as tier 1 Condition by the CDC, multiple KIs said no one wants to address it. To increase awareness, KIs wanted a champion who can advocate for legislative changes and public health attention. This may involve a partnership with politicians, payers, advocacy groups, or public health groups."[T]he disease states in which this has worked, there has been a champion that has worked behind the scenes, often maybe somebody that's a wealthy person that is able to influence lawmakers and in some cases like blind items have been inserted into legislation to make this happen." (KI10, research/advocacy, 15 years experience)

One KI in research/advocacy felt that the FH screening framework should be chosen based on the amount of public health support in that area, as well as the resources that are available for a program. This may also include the support from employers, payers, and advocacy groups in the area. Ultimately, public health support was thought to be beneficial to bring more providers into the fold, integrate changes at a broad systems level, and increase effectiveness of FH screening efforts. "I think it has to be a public health issue...our professional society guide- 
lines aren't working. Clearly the issue is too big, too common, there's too many provider practice variability issues...we should be thinking about ways to nationalize, you know an approach to FH because clearly, it's not working right now." (KI7, research/advocacy, 10 years experience)

\section{Research}

Some public health KIs stated that providers and public health departments were reluctant to adopt screening until there was more supporting research. "I think I would need to know a little more information about... how it would compare to some of the cancer screening programs in terms of number of individuals identified and potential impact on treatment and reduction of events um before I could [support screening]. Because it is a somewhat high bar to me, and there could potentially be a lot of costs associated with it - especially if we want to make sure that we include an equity component [for] the people that don't have access to this." (KI3, public health, 20 years experience)

KIs who participated in research did not mention a lack of research as a barrier to adopting FH screening. Public health KIs mentioned a need for research into the timing and safety of statin use in children, the cholesterol threshold to classify positive cases, the false-negative rate, and the false-positive rate. Overall, informants desired research to support the maintenance of a screening program.

\section{Implementation}

KIs recognized that it was difficult to implement an $\mathrm{FH}$ screening program, but many were interested in current pilot programs or participating in new screening programs. Support from guidelines to consistently apply FH screening was central to successful implementation.

\section{Guidelines}

Guidelines were identified by all KI groups as necessary for consistent implementation of FH screening. KIs cited issues with guideline complexity and length. KIs said that differing recommendations are confusing and specifically mentioned the differing recommendations between the USPSTF and the NHLBI/AAP. AHA/ACC guidelines were not mentioned. Of those who were familiar with guidelines, KIs preferred the NHLBI/AAP guidelines, but public health KIs said that these guidelines should be simplified, disseminated, and supported by public health to increase their implementation in clinics.

\section{Methodology}

KIs agreed that screening should be a cholesterol blood draw due to its high sensitivity, low cost, and broad availability. KIs suggested coordinating screening with other blood draws using nonfasting samples. The number of recommended cholesterol screens varied between KIs: with some suggesting multiple draws due to the variability in cholesterol levels. Alongside cholesterol screening, multiple KIs mentioned the importance of family history and genetic testing to differentiate true- and false-positive cases, clarify borderline cholesterol levels, and streamline cascade screening. KIs understood that sensitivity for genetic testing is limited, and most KIs suggested that genetic testing should include genetic counseling, alongside genetics outreach to support primary care. One KI suggested primary care could provide genetic testing to increase access. Many KIs were concerned about the availability of genetic testing services and the high cost; however, one KI stated the trend of lowering costs for genetic testing has created more availability. "I guess you feel bad making people go through genetic testing, which is expensive, and if it's not going to change how we treat them, I don't understand why we would need, you know, don't make them pay for this. It's not going to change how we do any of our treatment with them...It's selfish." (KI12, direct healthcare, 4.5 years experience)

Two KIs said that the inclusion of genetic testing in guidelines is necessary, and one KI mentioned the ethics of genetic testing in children as a concern."The ethics of genetic testing in kids I would say is still evolving, and right now I feel in most cases the advisory is against genetic testing in children. So I know it exists, but I don't view why they use the practice...I think it can still be treated appropriately without defining it with a genetic test which may or may not be something that a child wants to know when they're older." (KI16, direct healthcare, 15 years experience)

Many KIs mentioned the benefit of genetic testing in cascade screening, but insurance, access to healthcare services, and poor family communication were a deterrent for cascade screening. To increase availability of services, one KI suggested genetic testing videos, improved workflows with administrative support, and telehealth options.

\section{Cascade Screening}

KIs across groups agreed that cascade screening should be included in a screening program to find affected relatives with FH. KIs felt that it is the patient's responsibility to contact relatives to maintain patient autonomy and reduce provider burden. The provider's role should be to support the family in this by offering information and 
resources to share with relatives. A few KIs mentioned low awareness of cascade screening in primary care and the need for genetics outreach. "I think it's safe to say that from like a pediatric provider perspective they're thinking about that kid's particular risk, they're not thinking about it like a genetic counselor where...that could have implications for siblings, for parents or for whoever." (KI4, public health, 18.5 years experience)

Some KIs spoke about the success of cascade screening in single-payer healthcare programs and the challenges of integration of cascade screening into the US healthcare system. "W]e have not demonstrated an effective cascade screening methodology that is effective in terms of getting family members taken care of, and I think that's a big challenge. We know it can get done, it will work. Figuring out how to do it in our US healthcare system has yet to be proven." (KI8, research/advocacy, $60+$ years experience)

\section{Maintenance}

KIs felt that consistent funding apart from grants are important for maintenance of an FH screening program. Communication, coordination, and consistency of screening efforts over time was also suggested.

\section{Cost and Funding of Screening}

Public health KIs said cost-benefit analyses are useful to secure consistent funding. Because allocation of funding is dependent on the impact of each dollar spent, outcomes data are needed to support the allocation of funding for $\mathrm{FH}$ screening as opposed to other programs. One public health KI mentioned a "pay-for-performance" model focusing on preventative healthcare. Research/advocacy KIs had differing opinions about the financial sustainability of screening: one said universal screening was too costly relative to a more targeted screening approach, while another said universal screening was affordable when lab contracts were made to reduce the cost of screening. One direct healthcare KI suggested judicious use of specialist care and genetic testing in the interest of keeping costs low.

Funding was suggested through many different means, but state and federal funding was mentioned by all KI groups. State funding was recommended by most KIs, which could be supported through block grants or professional licensing fees. KIs in public health and research/ advocacy suggested individualized funding based on the state alongside legislative funding, which could be allocated to the CDC, Health Resources and Services Administration (HRSA), or regional collaborative groups. KIs in public health and direct healthcare anticipated that an $\mathrm{FH}$ legislative or advocacy champion would need to catalyze the monetary diversion from other programs. Nearly half of KIs suggested funding through grants, but it was recognized that they are short term and in short supply. All KI groups mentioned funding sourced from advocacy groups or individual donors. Over a quarter of KIs suggested billable services should fund the program.

\section{Consistency of Delivery}

KIs felt that increased coordination of screening efforts was needed to reliably deliver FH screening. Ongoing communication between public health, researchers, advocacy, and providers was viewed as crucial to maintain a screening program. This would include current screening efforts, data collection, and opportunities for improvement. "But um there isn't a forum... whether the CDC hosts it or the FH foundation or somebody, even if it was just an every-other-month conference call about brainstorming ideas and trying new things." (KI1, public health, 37 years experience)

\section{Discussion}

Diverse perspectives of stakeholders are necessary to address FH screening barriers. Stakeholder interest and engagement in FH screening was globally supportive of an FH screening program. Using the RE-AIM implementation framework, strategies emerged to approach screening through a varied, combinatory set of methods dependent on the population, setting, healthcare system, available resources, and public health support.

An FH screening program is highly dependent on healthcare providers, and there is wide variability in pediatricians' knowledge of guidelines and screening practices $[27-29,44]$. This study found similar variability amongst providers, but variability in practices and opinions expanded across public health, research, and advocacy KIs as well. The breadth of inconsistent practices and opinions has led to scattered FH-related healthcare and necessitates stakeholders to come together to address the issue, including professional organizations such as the AAP and USPSTF, advocacy groups such as the FH Foundation, payers, politicians, economists, and public health groups (Fig. 2). Adoption of FH screening will rely on multiple stakeholders working in concert. While each stakeholder has independent abilities and foci, there is shared responsibility to clearly outline $\mathrm{FH}$ screening for providers, or adoption of a consistent FH screening program will continue to falter. 
Fig. 2. Suggested steps to improve FH screening consistency. There is variability in $\mathrm{FH}$ screening guidelines and practices across the USA, within regions and within institutions. At each of these levels, there are steps that can be taken to reduce this variability. Increased consistency can be achieved by engaging stakeholders across domains (professional organizations, advocacy groups, payers, politicians, economists, and public health groups) to provide $\mathrm{FH}$ screening program guidance to regions. Regions can then tailor screening based on the amount of support they have and regional characteristics. Last, institutions within these regions can be supported to encourage individual providers to screen. FH, familial hypercholesterolemia.

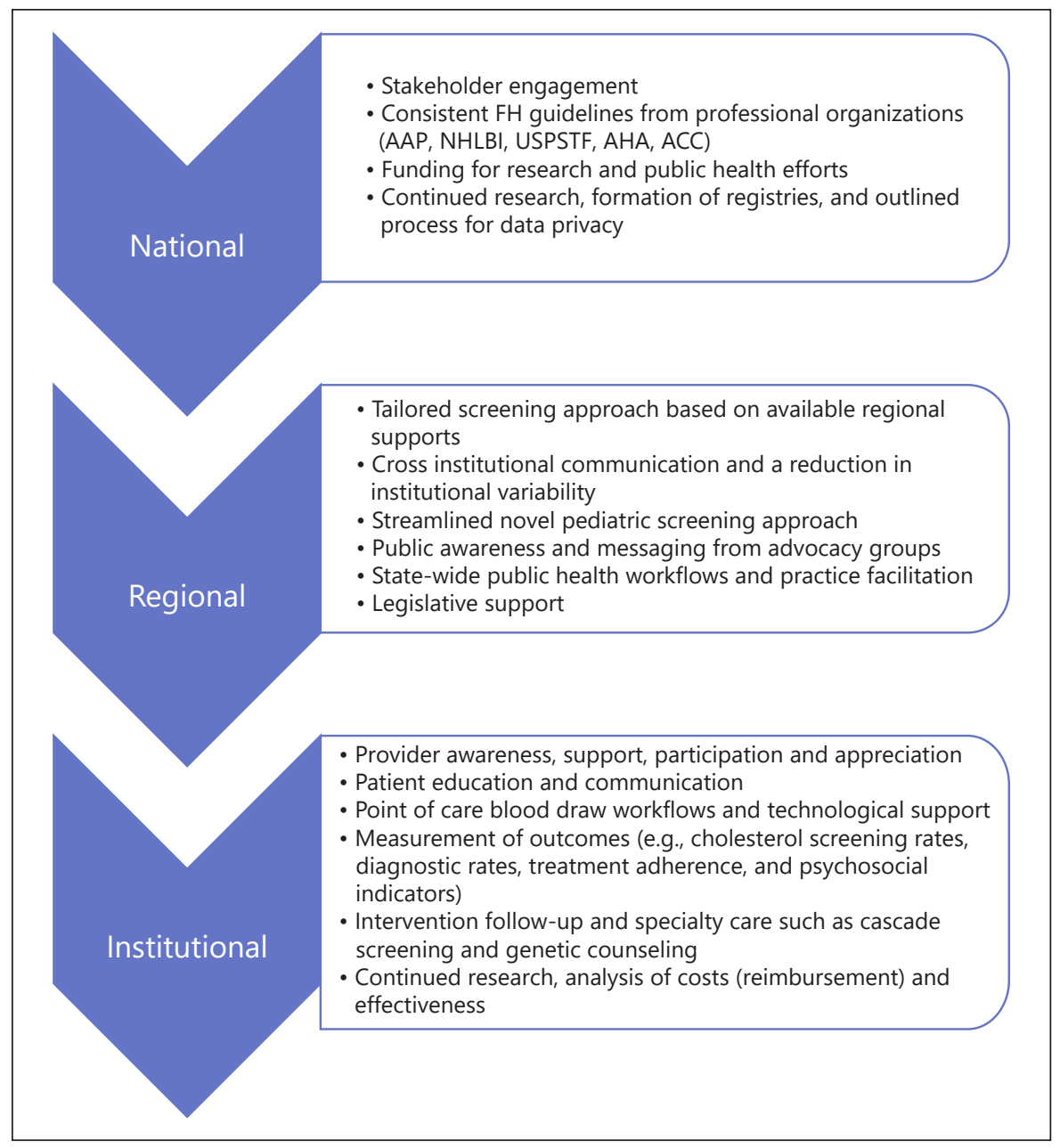

To ensure there is consistent and equitable patient access to FH screening, a diverse yet coordinated approach to FH screening is required. According to our study and others, the primary populations to screen should be children and young adults in accordance with the AHA/ACC guidelines, and bringing the USPSTF's guidelines in-line with other organizations will likely reduce provider confusion and improve the consistency of FH screening [11, $12,45]$. Studies on statin use in children are addressing the USPSTF concern for lack of long-term data [46]. If current guidelines continue to be inconsistently implemented, FH should be considered for a population-level screening program. Newborn screening has been discussed for many conditions including FH; however, there are significant technical limitations to using cholesterol screening in newborns. A novel screening program coupling various conditions, vaccines, and tests later in childhood may be more feasible. The benefits of early detec- tion and treatment should be considered alongside additional studies on the feasibility of FH screening programs, technical limitations, and harms of screening. Maximizing efficiency and likelihood of completion needs to be the target for FH screening.

FH screening effectiveness will be most effectively measured by using the currently successful CASCADEFH registry, but with the caveat that additional capabilities and state-wide registries may supplement the current database $[47,48]$. Global collaborations to create an FH registry have been explored by the European Atherosclerosis Society FH Studies Collaboration (EAS-FHSC) with the goal of informing health policy and generating largescale data on the FH burden worldwide [49]. Limitations to this approach include difficulties implementing the platform across clinics, provider education, data management and cleaning, and cost of data warehousing. The widespread use of aggregated registry data and dissemi- 
nation of research results between public health, research/ advocacy, and direct healthcare will help maintain and improve $\mathrm{FH}$ screening over time.

Regionally, whether that be divided as large geographic areas, states, or counties, there needs to be assistance in translating national efforts to institutions who will implement the screening. As each region has individual patient demographics, provider availability, funds, care facilities, and density, the national efforts will be tailored to the region. This will involve streamlining the screening practices from guidelines into functioning systems that can reduce institutional variability. Doing so will require the coordination of multiple systems, involvement of advocacy groups, and public awareness campaigns. Ensuring consistency will require state-level public health workflows, practice facilitation, and oversight to address health disparities. Last, legislative support to ensure its ongoing success.

On the institutional level, barriers to provider implementation from this study were consistent with other research citing barriers in EMR integration, physician discomfort with genomic medicine, inadequate reimbursement, and lack of leadership engagement [50, 51]. Provider comfort with genomic medicine can be increased via educational materials, meetings, and outreach events [50]. To reduce provider burden, automated provider prompts (e.g., cholesterol screening for all 9- to 11-year-olds) can increase consistent FH screening, as demonstrated by the EMR SEARCH algorithm created at Mayo Clinic and the FIND FH algorithm created by the FH Foundation [52-54]. As $87 \%$ of physicians use an EMR, EMRs can also help connect providers to existing resources including tools for diagnosis, management, and registries.

There was general agreement between KIs in methodology and intervention agents. With the addition of genetic testing and cascade screening, suspected cases of FH can be clarified and family members identified $[11,55]$. Genetic testing has been shown to be effective in employing cascade screening and has been supported by multiple organizations including the $\mathrm{FH}$ Foundation and the $\mathrm{Na}$ tional Lipid Association [33, 56, 57]. With wide support for cascade screening across KIs, the lack of provider awareness and comfort with genetic testing may be improved by genetics outreach around clinical utility of genetic testing, family dynamics and geographic dispersion, healthcare literacy, privacy concerns, cost and insurance coverage, and access to care $[26,58]$.

Stable funding, coordination, and communication between stakeholders is essential to maintain FH screening. As programs are adopted and implemented, unforeseen issues can be reliably addressed through already-established lines of communication such as conference calls between public health, researchers, advocacy, and providers.

This study utilized KIs who were recruited by study researchers, and the KIs who agreed to be interviewed are likely motivated and supportive of FH screening implementation. Eleven KIs did not respond to the recruitment emails, limiting our ability to draw conclusions about nonparticipation. KIs who did not have experience in $\mathrm{FH}$ but had expertise in screening or public health were intentionally included to bring alternative and diverse perspectives. Interviews reached theme saturation after 12 were completed. Some interviews were time constrained, which prevented several KIs from completing all interview questions. The structure of the interviews was semistructured to allow KIs to elaborate on their concerns and interests, but also allowed for interview variability and biased responses. Interviewer biases were in favor of genetic testing due to her professional role as a genetic counseling student, and the last author (H.Z.) has research funding for an $\mathrm{FH}$ cascade screening intervention. Some KIs were aware of the interviewer's and H.Z.'s demographics, which may have influenced their responses. The questions were designed and coded using the REAIM framework, which biases the topics of discussion and may hinder the recognition of other factors in $\mathrm{FH}$ screening. Last, the directed content analysis has a tendency to recognize supportive evidence rather than nonsupportive evidence of the RE-AIM framework [42].

KIs communicated a need for attention and action in FH screening. Many KIs mentioned the need for a champion who can foster legislative changes and the need to bring together stakeholders. To address the need for more perspectives on the topic and at a regional- or state-level, politicians, the general public, health economists, and payers may bring beneficial perspectives to the implementation of FH screening. To make use of the extensive $\mathrm{FH}$-related research, dissemination to stakeholders must be improved. Economic analyses can support the most appropriate screening framework. To increase consistent delivery of FH screening, public health-supported practice facilitation within primary care clinics and coordinated blood draws should be explored. Population-level screening feasibility, technical explorations, and harms of screening at this age should be investigated. The shift from discussion to action will be the most beneficial step toward FH diagnosis and treatment, and implementation of a program will likely be welcomed by providers if it is accompanied by appropriate public health and advocacy supports. 


\section{Acknowledgements}

We would like to thank Krista Redlinger-Grosse, PhD, and Ellen Demerath, $\mathrm{PhD}$, for their participation on the graduate committee and revisions of this manuscript. We would also like to acknowledge Hannaan Shire and Sonia Ehrlich for their work in transcribing the interviews. Last, we would like to thank the University of Minnesota Genetic Counseling Program for their support in completing this research.

\section{Statement of Ethics}

The University of Minnesota Institutional Review Board (IRB) determined that this research (STUDY00003854) is not research involving human subjects as defined by the Department of Health and Human Services (DHHS) and the Food and Drug Administration (FDA) regulations and is therefore IRB exempt.

\section{Conflict of Interest Statement}

The authors have no conflicts of interest to declare.

\section{Funding Sources}

Last author (H.Z.) has research funding for FH cascade screening intervention research.

\section{Author Contributions}

This study was conceived by H.Z. and designed by all authors. Data were collected and analyzed by D.M. A.G. and H.Z. audited the data analysis. D.M. wrote the manuscript, which was edited by all authors.

\section{References}

1 Rader DJ, Cohen J, Hobbs HH. Monogenic hypercholesterolemia: new insights in pathogenesis and treatment. J Clin Invest. 2003 Jun 15 [cited 2018 Apr 29];111(12):1795-803.

2 Goldberg AC, Hopkins PN, Toth PP, Ballantyne CM, Rader DJ, Robinson JG, et al. Familial hypercholesterolemia: screening, diagnosis and management of pediatric and adult patients. J Clin Lipidol. 2011 Jun [cited 2018 Apr 29];5(3):S1-8

3 Knowles JW, O'Brien EC, Greendale K, Wilemon K, Genest J, Sperling LS, et al. Reducing the burden of disease and death from familial hypercholesterolemia: a call to action. Am Heart J. 2014 Dec [cited 2018 Apr 29];168(6): $807-11$.

4 Akioyamen LE, Genest J, Shan SD, Reel RL, Albaum JM, Chu A, et al. Estimating the prevalence of heterozygous familial hypercholesterolaemia: a systematic review and metaanalysis. BMJ Open. 2017 Sep 1 [cited 2018 Mar 25];7(9):e016461.

5 Agabiti Rosei E, Salvetti M. Management of hypercholesterolemia, appropriateness of therapeutic approaches and new drugs in patients with high cardiovascular risk. High Blood Press Cardiovasc Prev. 2016 Sep [cited 2018 Mar 25];23(3):217-30.

6 Neil HA, Hammond T, Huxley R, Matthews DR, Humphries SE. Extent of underdiagnosis of familial hypercholesterolaemia in routine practice: prospective registry study. BMJ. 2000 Jul 15 [cited 2018 Apr 22];321(7254): 148.

7 Wiegman A, Gidding SS, Watts GF, Chapman MJ, Ginsberg HN, Cuchel M, et al. Familial hypercholesterolaemia in children and adolescents: gaining decades of life by optimizing detection and treatment. Eur Heart J. 2015 Sep 21 [cited 2018 Feb 17];36(36):2425-37.

8 Nordestgaard BG, Chapman MJ, Humphries SE, Ginsberg HN, Masana L, Descamps OS, et al. Familial hypercholesterolaemia is underdiagnosed and undertreated in the general population: guidance for clinicians to prevent coronary heart disease: consensus statement of the European Atherosclerosis Society. Eur Heart J. 2013 Dec 1 [cited 2018 Apr 29]; 34(45):3478-90a.

9 Wilson JMG, Jungner G. The principles and practice of screening for disease. World Heal Organ. 1966 [cited 2018 Mar 25].

10 CDC Office of Public Health Genomics. Genomic tests by levels of evidence: tier 1 genomic and family health history applications are recommended for clinical use by evidence-based panels based on a systematic review of analytic validity. Clinical validity and utility for specific clinical scenarios [Internet]. 2013 [cited 2018 Nov 2].

11 NHBLI. Expert panel on integrated guidelines for cardiovascular health and risk reduction in children and adolescents: summary report. Pediatrics. 2011 Dec 1 [cited 2018 Nov 1]; 128(Suppl 5):S213-56.

12 Grundy SM, Stone NJ, Bailey AL, Beam C, Birtcher KK, Blumenthal RS, et al. 2018 AHA/ ACC/AACVPR/AAPA/ABC/ACPM/ADA/ AGS/APhA/ASPC/NLA/PCNA guideline on the management of blood cholesterol: a report of the American College of Cardiology/ American Heart Association Task Force on Clinical Practice Guidelines [Internet]. In: Circulation. Philadelphia, PA: Lippincott Williams and Wilkins; 2019 [cited 2020 Oct 29]. Vol. 139; p. E1082-143.

13 National Clinical Guideline Centre. Familial hypercholesterolaemia: identification and management|Guidance and guidelines. NICE. 2017 [cited 2018 Nov 1].

14 Marks D, Wonderling D, Thorogood M, Lambert H, Humphries SE, Neil HA. Screening for hypercholesterolaemia versus case finding for familial hypercholesterolaemia: a systematic review and cost-effectiveness analysis. Health Technol Assess. 2000 [cited 2018 Mar 25];4(29):1-123.

15 U.S. Preventative Services Task Force. Final recommendation statement: lipid disorders in children and adolescents: screening. Recomm Prim Care Pract. 2016 Sep [cited 2018 Nov 2].

16 Weng SF, Kai J, Andrew Neil H, Humphries SE, Qureshi N. Improving identification of familial hypercholesterolaemia in primary care: derivation and validation of the familial hypercholesterolaemia case ascertainment tool (FAMCAT). Atherosclerosis. 2015 Feb [cited 2018 Apr 29];238(2):336-43.

17 Bhatnagar D. Diagnosis and screening for familial hypercholesterolaemia: finding the patients, finding the genes. Ann Clin Biochem. $2006 \mathrm{Nov}$ 1 [cited 2018 Mar 25];43(Pt 6):441-56.

18 Taylor A, Wang D, Patel K, Whittall R, Wood $\mathrm{G}$, Farrer M, et al. Mutation detection rate and spectrum in familial hypercholesterolaemia patients in the UK pilot cascade project. Clin Genet. 2010 Jun [cited 2018 Mar 25];77(6): 572-80.

19 Bourbon M, Alves AC, Alonso R, Mata N, Aguiar P, Padró T, et al. Mutational analysis and genotype-phenotype relation in familial hypercholesterolemia: the SAFEHEART registry. Atherosclerosis. 2017 Jul [cited 2019 Apr 24];262:8-13.

20 Umans-Eckenhausen MA, Defesche JC, Sijbrands EJ, Scheerder RL, Kastelein JJ. Review of first 5 years of screening for familial hypercholesterolaemia in the Netherlands. Lancet. 2001 Jan 20 [cited 2019 Apr 24];357(9251):165-8.

21 Migliara G, Baccolini V, Rosso A, D’Andrea E, Massimi A, Villari P, et al. Familial hypercholesterolemia: a systematic review of guidelines on genetic testing and patient management. Front Public Health. 2017 Sep 25 [cited 2018 Apr 29];5:252.
Implementation Framework for Overcoming Underdiagnoses of FH 
22 Hadfield SG, Horara S, Starr BJ, Yazdgerdi S, Marks D, Bhatnagar D, et al. Family tracing to identify patients with familial hypercholesterolaemia: the second audit of the Department of Health Familial Hypercholesterolaemia Cascade Testing Project. Ann Clin Biochem. 2009 Jan 1 [cited 2018 Mar 25];46(Pt 1):2432.

23 Ademi Z, Watts GF, Pang J, Sijbrands EJ, van Bockxmeer FM, O'Leary P, et al. Cascade screening based on genetic testing is cost-effective: evidence for the implementation of models of care for familial hypercholesterolemia. J Clin Lipidol. 2014 [cited 2018 Feb 17]; 8(4):390-400.

24 Hardcastle SJ, Legge E, Laundy CS, Egan SJ, French R, Watts GF, et al. Patients' perceptions and experiences of familial hypercholesterolemia, cascade genetic screening and treatment. Int J Behav Med. 2015 Feb 1 [cited 2018 Mar 24];22(1):92-100.

25 Muir LA, George PM, Whitehead L. Using the experiences of people with familial hypercholesterolaemia to help reduce the risk of cardiovascular disease: a qualitative systematic review. J Adv Nurs. 2012 Sep [cited 2018 Mar 24];68(9):1920-32.

26 Roberts MC, Dotson WD, DeVore CS, Bednar EM, Bowen DJ, Ganiats TG, et al. Delivery of cascade screening for hereditary conditions: a scoping review of the literature. Health Affairs. 2018 May [cited 2018 Nov 2]; 37(5):801-8.

27 Zimmerman J, Duprez D, Veach PM, Zierhut HA. Barriers to the identification of familial hypercholesterolemia among primary care providers. J Community Genet. 2019 Apr 11 [cited 2019 Apr 24];10(2):229-36.

28 Dixon DB, Kornblum AP, Steffen LM, Zhou $\mathrm{X}$, Steinberger J. Implementation of lipid screening guidelines in children by primary pediatric providers. J Pediatr. 2014 Mar 1 [cited 2018 Feb 17];164(3):572-6.

29 Allen-Tice C, Steinberger J, Murdy K, Zierhut H. Pediatric cholesterol screening practices in 9- to 11-year-olds in a large midwestern primary care setting. J Clin Lipidol. 2020;14(2): 224-30.

30 Wonderling D, Umans-Eckenhausen MA, Marks D, Defesche JC, Kastelein JJ, Thorogood M. Cost-effectiveness analysis of the genetic screening program for familial hypercholesterolemia in the Netherlands. Semin Vasc Med. 2004 Mar 22 [cited 2018 Mar 25]; 4(1):97-104.

31 Kerr M, Pears R, Miedzybrodzka Z, Haralambos K, Cather M, Watson M, et al. Cost effectiveness of cascade testing for familial hypercholesterolaemia, based on data from familial hypercholesterolaemia services in the UK. Eur Heart J. 2017 Jun 14 [cited 2018 Apr 29]; 38(23):1832-9.

32 Rosso A, Pitini E, D’Andrea E, Massimi A, De Vito C, Marzuillo C, et al. The cost-effectiveness of genetic screening for familial hypercholesterolemia: a systematic review. Ann Ig. 2017 [cited 2018 Apr 29];29(5):464-80.
33 Alver M, Palover M, Saar A, Läll K, Zekavat SM, Tõnisson N, et al. Recall by genotype and cascade screening for familial hypercholesterolemia in a population-based biobank from Estonia. Genet Med. 2018 Oct 1 [cited 2019 Apr 25].

34 Klančar G, Grošelj U, Kovač J, Bratanič N, Bratina N, Trebušak Podkrajšek K, et al. Universal screening for familial hypercholesterolemia in children. J Am Coll Cardiol. 2015 Sep 15 [cited 2018 Feb 10];66(11):1250-7.

35 Florentin M, Kostapanos MS, Elisaf MS, Liberopoulos EN. Prevalence, identification, and scouting for familial hypercholesterolaemia including registries. Curr Pharm Des. 2018 Oct 9 [cited 2018 Oct 29];24(31):3605

36 Umans-Eckenhausen MA, Defesche JC, Sijbrands EJ, Scheerder RL, Kastelein JJ. Review of first 5 years of screening for familial hypercholesterolaemia in the Netherlands. Lancet. 2001 Jan 20 [cited 2018 Apr 29];357(9251): 165-8.

37 Morris JK, Wald DS, Wald NJ. The evaluation of cascade testing for familial hypercholesterolemia. Am J Med Genet A. 2012 Jan [cited 2019 Apr 25];158A(1):78-84.

38 Glasgow RE, Estabrooks PE. Pragmatic applications of RE-AIM for health care initiatives in community and clinical settings. Prev Chronic Dis. 2018 [cited 2019 Apr 7];15:E02.

39 Holtrop JS, Rabin BA, Glasgow RE. Qualitative approaches to use of the RE-AIM framework: rationale and methods. BMC Health Serv Res. 2018 [cited 2019 Apr 7];18(1):177.

40 Gaglio B, Shoup JA, Glasgow RE. The REAIM framework: a systematic review of use over time. Am J Public Health. 2013 Jun [cited 2019 Apr 7];103(6):e38-46.

41 Patton MQ. Qualitative research \& evaluation methods. 3rd ed. Thousand Oaks, California: Sage Publications; 2002.

42 Hsieh HF, Shannon SE. Three approaches to qualitative content analysis. Qual Health Res. 2005 [cited 2019 Apr 6];15(9):1277.

43 Glasgow RE, Vogt TM, Boles SM. Evaluating the public health impact of health promotion interventions: the RE-AIM framework. Am J Public Health. 1999 Sep [cited 2019 Apr 7]; 89(9):1322-7.

44 de Ferranti SD, Rodday AM, Parsons SK, Cull WL, O'Connor KG, Daniels SR, et al. Cholesterol screening and treatment practices and preferences: a survey of United States pediatricians. J Pediatr. 2017 Jun [cited 2019 Apr 23];185:99-e2.

45 Committee on Practice and Ambulatory Medicine, Bright Futures Periodicity Schedule Workgroup. 2017 recommendations for preventive pediatric health care. Pediatrics. 2017 Apr 1 [cited 2018 Nov 1];139(4):e20170254.

46 Luirink IK, Wiegman A, Kusters DM, Hof MH, Groothoff JW, De Groot E, et al. 20-Year follow-up of statins in children with familial hypercholesterolemia. N Engl J Med. 2019 Oct 17 [cited 2020 Apr 9];381(16):1547-56.

47 O’Brien EC, Roe MT, Fraulo ES, Peterson ED, Ballantyne CM, Genest J, et al. Rationale and design of the familial hypercholesterolemia foundation CAscade SCreening for Awareness and DEtection of Familial Hypercholesterolemia registry. Am Heart J. 2014 Mar [cited 2018 Nov 2];167(3):342-9.e17.

48 deGoma EM, Ahmad ZS, O'Brien EC, Kind I, Shrader P, Newman CB, et al. Treatment gaps in adults with heterozygous familial hypercholesterolemia in the United States: data from the CASCADE-FH registry. Circ Cardiovasc Genet. 2016 Jun [cited 2018 Nov 2]; 9(3):240-9.

49 Vallejo-Vaz AJ, Vallejo-Vaz AJ, Akram A, Kondapally Seshasai SR, Cole D, Watts GF, et al. Pooling and expanding registries of familial hypercholesterolaemia to assess gaps in care and improve disease management and outcomes: rationale and design of the global EAS Familial Hypercholesterolaemia Studies Collaboration. Atheroscler Suppl. 2016 Dec [cited 2018 Feb 10];22:1-32.

50 Sperber NR, Carpenter JS, Cavallari LH, J Damschroder LL, Cooper-DeHoff RM, Denny JC, et al. Challenges and strategies for implementing genomic services in diverse settings: experiences from the Implementing GeNomics In pracTicE (IGNITE) network. BMC Med Genomics. 2017 May 22;10(1):35.

51 Zebrowski AM, Ellis DE, Barg FK, Sperber NR, Bernhardt BA, Denny JC, et al. Qualitative study of system-level factors related to genomic implementation. Genet Med. 2018 Nov 23 [cited 2019 Apr 25].

52 Safarova MS, Liu H, Kullo IJ. Rapid identification of familial hypercholesterolemia from electronic health records: the SEARCH study. J Clin Lipidol. 2016 Sep [cited 2018 Nov 2]; 10(5):1230-9.

53 Jamoom E, Yang N. Table of electronic health record adoption and use among office-based physicians in the U.S., by state: 2015 National Electronic Health Records Survey. [Internet]. 2016 [cited 2019 Apr 25].

54 The FH Foundation. FIND FH [Internet]. [cited 2019 Apr 29]. Available from: https:// thefhfoundation.org/find-fh.

55 Alonso R, Perez de Isla L, Muñiz-Grijalvo O, Diaz-Diaz JL, Mata P. Familial hypercholesterolaemia diagnosis and management. Eur Cardiol Rev. 2018 Aug [cited 2018 Oct 29]; 13(1):14

56 Sturm AC, Knowles JW, Gidding SS, Ahmad ZS, Ahmed CD, Ballantyne CM, et al. Clinical genetic testing for familial hypercholesterolemia: JACC Scientific Expert Panel. J Am Coll Cardiol. 2018 Aug 7 [cited 2019 Apr 23]; 72(6):662-80.

57 Hendricks-Sturrup R, Lu C. Understanding implementation challenges to genetic testing for familial hypercholesterolemia in the United States. J Pers Med. 2019 Feb 1 [cited 2019 Apr 29];9(1):9.

58 Knowles JW, Rader DJ, Khoury MJ. Cascade screening for familial hypercholesterolemia and the use of genetic testing. JAMA. $2017 \mathrm{Jul}$ 25 [cited 2019 Apr 24];318(4):381. 